\title{
Quorum signaling and sensing by nontypeable Haemophilus influenzae
}

\author{
W. Edward Swords* \\ Department of Microbiology and Immunology, Wake Forest University Health Sciences, Winston-Salem, NC, USA
}

\section{Edited by:}

John S. Gunn, The Ohio State

University, USA

Reviewed by:

Margaret E. Bauer, Indiana

University School of Medicine, USA

Arnold L. Smith, Seattle Children's

Research Institute, USA

*Correspondence:

W. Edward Swords, Department of

Microbiology and Immunology,

Wake Forest University Health

Sciences, 575 North Patterson Ave,

2E-034 Wake Forest Biotech Place,

Winston-Salem, NC 27101, USA.

e-mail:wswords@wakehealth.edu
Quorum signals are diffusible factors produced by bacteria that coordinate communal responses. For nontypeable Haemophilus influenzae (NTHi), a series of recent papers indicate that production and sensing of quorum signals are determinants of biofilm formation/maturation and persistence in vivo. In this mini-review I will summarize the current knowledge about quorum signaling/sensing by this organism, and identify specific topics for additional study.

Keywords: biofilm, Haemophilus influenzae, otitis media, quorum signals, chronic

\section{INTRODUCTION}

Bacterial quorum sensing involves community-dependent regulation of bacterial gene expression by means of soluble signal molecules that are released in a density-dependent manner (Miller and Bassler, 2001; Henke and Bassler, 2004; Waters and Bassler, 2005). These signaling molecules are chemically diverse, and include the so-called competence factor peptides of pneumocci and other gram-positive bacteria, homoserine lactones, and derivatives of the bacterial metabolic by-product dihydroxypentanedione (DPD), which is also referred to as autoinducer-2 (AI-2). Because production of DPD is widely conserved among bacterial species, it has been referred to as an interspecies quorum signal (Surette et al., 1999).

Quorum sensing has been shown to coordinate group activities among bacterial populations, including formation and maturation of biofilm communities (Parsek and Singh, 2003; Henke and Bassler, 2004; Irie and Parsek, 2008; Shrout et al., 2011). In this mini-review, I discuss the current knowledge about quorum signaling and sensing in nontypeable Haemophilus influenzae (NTHi), and highlight potential areas for future study.

\section{RELATIONSHIP BETWEEN DPD (AI-2) QUORUM SIGNALS AND VIRULENCE}

The current published work concerning NTHi quorum signaling and sensing is summarized in Table 1. Quorum signaling for this species was first suggested by presence of the luxS genetic determinant of DPD production in the H. influenzae Rd genomic sequence (Surette et al., 1999). Later, Daines and colleagues showed that numerous NTHi strains produced quorum signal as detected by the Vibrio harveyi bioluminescence assay (Daines et al., 2005). Also in this study, luxS transcript levels were shown to be increased during infection of epithelial cells, and mutants lacking $\operatorname{lux} S$ were also shown to retain the capacity to form biofilms, have increased invasion of epithelial cells, and cause more severe otitis media disease in the chinchilla infection model.

Later work from our laboratory expanded on this work to show that while isogenic NTHi mutants lacking luxS do retain the capacity to form biofilms, quantitative assessment of the biofilm structure by confocal microscopic analysis showed significantly reduced thickness and density, which was restored by complementation or by co-culture with the parental strains (Armbruster et al., 2009). Chinchilla infection studies also revealed that luxS mutants caused a more acute, inflammatory infection and that long-term persistence of luxS mutants was significantly reduced as compared with the parental strain (Armbruster et al., 2009). These changes in biofilm were correlated with shifts in the lipooligosaccharide glycolipids on the bacterial surface, which had previously been shown by our group to promote biofilm maturation and persistence in vivo (Hong et al., 2007a,b).

More recently, the RbsB protein was shown to mediate uptake of DPD quorum signals for NTHi 86-028NP (Armbruster et al., 2011). RbsB is a periplasmic binding protein which functions as part of an $A B C$ transporter for ribose sugars (Park et al., 1999), and has been shown to function in AI-2 uptake in other bacterial species (Shao et al., 2007). Similar to luxS mutants, isogenic $r b s B$ mutants were demonstrated to produce biofilms with significantly reduced thickness and density as compared to the parental NTHi strain. These changes in biofilm were correlated with changes in the lipooligosaccharide content and a persistence defect in the chinchilla infection model (Armbruster et al., 2011). However, given the genomic diversity among NTHi strains, it is important to note that there is a strong possibility for other determinants of quorum signal uptake (or absence of RbsB) in other strains (Pereira et al., 2009). In support of this 
Table 1 | Current knowledge about quorum signaling/sensing in H. influenzae.

\begin{tabular}{ll}
\hline Finding & Reference \\
\hline $\begin{array}{l}\text { NTHi luxS mutants have increased invasion } \\
\text { NTHi luxS mutants cause acute otitis media } \\
\text { with greater inflammation }\end{array}$ & Daines et al., 2005 \\
Quorum signaling promotes NTHi biofilm & \\
maturation & Armbruster et al., 2009 \\
$\begin{array}{l}\text { Quorum signaling affects lipooligosaccharide } \\
\text { composition }\end{array}$ & Armbruster et al., 2009 \\
NTHi quorum signals affect M. catarrhalis & \\
RbsB is a determinant of quorum signal & Armbruster et al., 2010 \\
uptake for strain NTHi 86-028NP & Armbruster et al., 2011 \\
\hline
\end{tabular}

idea, examination of the 18 NTHi publicly accessible genomic sequences reveals that while the majority of strains (12/18) would be predicted to only have the Rbs system for uptake, orthologs of the Lsr system associated with quorum signal uptake and sensing in other bacterial species are found in $3 / 18$ strains; in one of these the Rbs transporter is not found. Notably, in 3/18 strains there were no predicted sequences for either transporter. Thus, the potential exists for significant mechanistic diversity in quorum signal uptake (and presumably, sensing) among different NTHi strains. We are currently addressing this important topic.

\section{INTERSPECIES QUORUM SIGNALING}

In addition to the impact of quorum signaling on NTHi biofilms, recent work has demonstrated that NTHi quorum signals may impact Moraxella catarrhalis, an opportunistic pathogen that inhabits many of the same host environments within the airway. Growth of $M$. catarrhalis within a polymicrobial biofilm with nontypeable $H$. influenzae was shown to promote antibiotic resistance and persistence within the chinchilla infection model (Armbruster et al., 2010). In support of quorum signaling as a mechanism for these effects on M. catarrhalis, there was no significant benefit in terms of antibiotic resistance or persistence in vivo in parallel experiments using an isogenic NTHi luxS mutant strain. Notably, no AI-2 quorum signal production was detected for any $M$. catarrhalis strain, and recent data analyzing a number of sequenced $M$. catarrhalis genomes show that none have a homologue for the luxS genetic determinant of this quorum signal (Davie et al., 2011). Moreover, M. catarrhalis bacteria had the capacity to take up purified DPD from culture supernatants, and addition of purified DPD to M. catarrhalis bacteria also promoted biofilm density and antibiotic resistance in vivo (Armbruster et al., 2010). Taken together, these results show that M. catarrhalis "eavesdrops" on NTHi quorum signals to coordinate its biofilm development. These results are consistent with epidemiologic data that indicate a significant correlation of $M$. catarrhalis with NTHi co-infection in clinical samples from patients with otitis media or other opportunistic airway infections (Pettigrew et al., 2008; Verhaegh et al., 2011).

\section{CONTROVERSIES, REMAINING OUESTIONS AND TOPICS FOR ADDITIONAL STUDY}

While present data clearly establish the importance of quorum signaling/sensing for some NTHi model strains, much remains to be learned on this topic and its relationship to virulence (summarized in Figure 1). The magnitude and kinetics of quorum signal production by different NTHi strains is not presently known, and there is a distinct possibility that some strains may not fit with the current knowledge that has mostly been derived from NTHi 86-028NP. As highlighted above, genomic analyses clearly indicate presence of other potential AI-2 transporters in some NTHi genomes; whether these strains have greater capacity for signal uptake (and presumably sensing) is a subject for additional study. All sequenced NTHi strains have homologs to the QseB/C twocomponent signaling system that mediates sensing of AI-2/DPD signals for some other bacterial species; the role of these factors in sensing of quorum signal by NTHi is not presently clear. Finally, generation of NTHi mutant strains in which AI-2 quorum signal may be artificially induced would be of great help not only in clarifying the direct linkage of quorum signal to biofilm formation/maturation but also in defining the consequences of quorum sensing for the bacterial population.

It should also be pointed out that the relevance of biofilms for NTHi infection is not universally accepted (Moxon et al., 2008). Criticisms of this concept have included the lack of a defined matrix component expressed exclusively during biofilm growth, observation of aggregated bacteria with some of the descriptive qualities associated with biofilm in plate cultures, and lack of evidence for a controlled development of a differentiated community within NTHi biofilms/aggregates. Certainly, there was at the time of this commentary a great need for better understanding of the process of biofilm development and the role(s) of biofilms in the persistence of NTHi bacteria in vivo. For example, prior to our demonstration of NTHi survival within neutrophil extracellular traps (NET; Hong et al., 2009; Juneau et al., 2011), it could have been plausible to consider the possibility that the surface-adherent bacteria, rather than persisting, were being killed. Likewise, the findings discussed above regarding role(s) for synthesis and uptake of quorum signal (Armbruster et al., 2009, 2011), and restoration of biofilm phenotype by addition

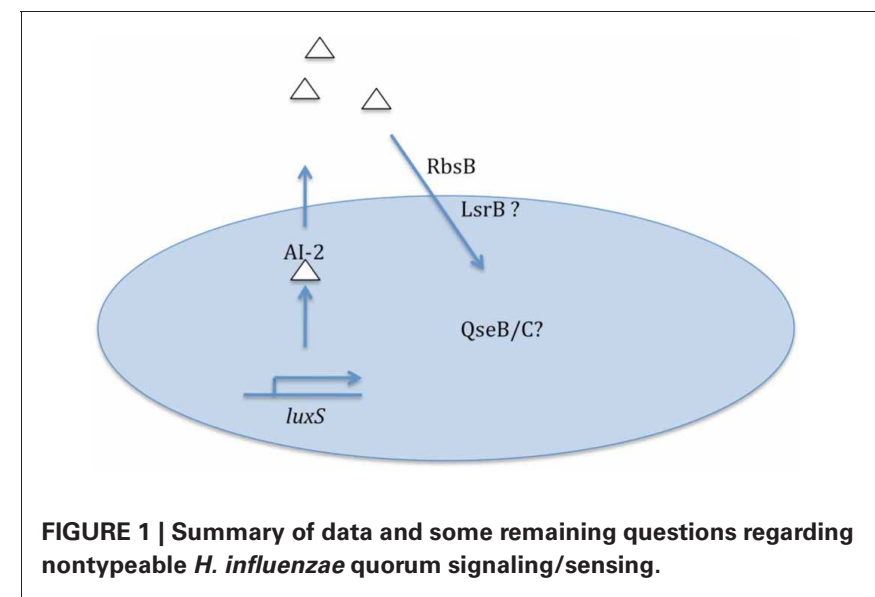


of culture supernatants or synthetic DPD to luxS mutants (Armbruster et al., 2009, 2011), provide additional evidence for coordinated development of a biofilm. It is also important to note work from other species indicating roles for nutrient composition of growth media in biofilm formation/maturation that can equal or even surpass that of quorum signaling/sensing (Shrout

\section{REFERENCES}

Armbruster, C., Byrd, M., Love, C., Juneau, R., Kock, N. D., and Swords, W. E. (2009). LuxS promotes biofilm maturation and persistence of nontypeable Haemophilus influenzae in experimental otitis media by modulation of lipooligosaccharide composition. Infect. Immun. 77, 4081-4091.

Armbruster, C. E., Hong, W., Pang, B., Weimer, K. E., Juneau, R. A., Turner, J., and Swords, W. E. (2010). Indirect pathogenicity of Haemophilus influenzae and Moraxella catarrhalis in polymicrobial otitis media occurs via interspecies quorum signaling. MBio 1, 102-110.

Armbruster, C. E., Pang, B., Murrah, K., Juneau, R. A., Perez, A. C., Weimer, K. E., and Swords, W. E. (2011). RbsB (NTHI-0632) mediates quorum signal uptake in nontypeable Haemophilus influenzae strain 86028NP. Mol. Microbiol. 82, 836-850.

Daines, D. A., Bothwell, M., Furrer, J., Unrath, W., Nelson, K., Jarisch, J., Melrose, N., Greiner, L., Apicella, M., and Smith, A. L. (2005). Haemophilus influenzae luxs mutants form a biofilm and have increased virulence. Microb. Pathog. 39, 87-96.

Davie, J. J., Earl, J., de Vries, S. P., Ahmed, A., Hu, F. Z., Bootsma, H. J., Stol, K., Hermans, P. W., Wadowsky, R. M., Ehrlich, G. D., Hays, J. P., and Campagnari, A. A. (2011). Comparative analysis and supragenome modeling of twelve Moraxella catarrhalis clinical isolates. BMC Genomics 12, 70.

Henke, J. M., and Bassler, B. L. (2004). Bassler, Bacterial social engagements. Trends Cell Biol. 14, 648-656.

Hong, W., Juneau, R., Pang, B., and Swords, W. E. (2009). Survival of bacterial biofilms within neutrophil extracellular traps promotes nontypeable Haemophilus influenzae persistence in the chinchilla model for otitis media. J. Innate Immun. 1, 215-224.

Hong, W., Mason, K., Jurcisek, J. A., Novotny, L. A., Bakaletz, L. O., and Swords, W. E. (2007a). Phosphorylcholine decreases early inflammation and promotes the establishment of stable biofilm communities of nontypeable Haemophilus influenzae strain 86-028NP in a chinchilla model of otitis media. Infect. Immun. 75, 958-965.

Hong, W., Pang, B., West-Barnette, S., and Swords, W. E. (2007b). Phosphorylcholine expression by nontypeable Haemophilus influen$z a e$ correlates with maturation of biofilm communities in vitro and in vivo. J. Bacteriol. 189, 8300-8307.

Irie, Y., and Parsek, M. R. (2008). Quorum sensing and microbial biofilms. Curr. Top. Microbiol. Immunol. 322, 67-84.

Juneau, R. A., Pang, B., Weimer, K. E., Armbruster, C. E., and Swords, W. E. (2011). Nontypeable Haemophilus influenzae initiates formation of neutrophil extracellular traps. Infect. Immun. 79, 431-438.

Miller, M. B., and Bassler, B. L. (2001). Quorum sensing in bacteria. Annu. Rev. Microbiol. 55, 165-199.

Moxon, E. R., Sweetman, W. A., Deadman, M. E., Ferguson, D. J., and Hood, D. W. (2008).

et al., 2006, 2011). This will surely be an important variable for additional study with regard to NTHi biofilms.

\section{ACKNOWLEDGMENTS}

Work in our laboratory is supported by funding from the NIH (DC007444, DC10051, and DC12205).

Haemophilus influenzae biofilms: hypothesis or fact? Trends Microbiol. $16,95-100$.

Park, Y., Cho, Y. J., Ahn, T., and Park, C. (1999). Molecular interactions in ribose transport: the binding protein module symmetrically associates with the homodimeric membrane transporter. $E M B O \mathrm{~J} .18$, $4149-4156$

Parsek, M. R., and Singh, P. K. (2003). Bacterial biofilms: an emerging link to disease pathogenesis. Annu. Rev. Microbiol. 57, 677-701.

Pereira, C. S., de Regt, A. K., Brito, P. H., Miller, S. T., and Xavier, K. B. (2009). Identification of functional LsrB-like autoinducer-2 receptors. J. Bacteriol. 191, 6975-6987.

Pettigrew, M. M., Gent, J. F., Revai, K., Patel, J. A., and Chonmaitree, T. (2008). Microbial interactions during upper respiratory tract infections. Emerging Infect. Dis. 14, 1584-1591.

Shao, H., James, D., Lamont, R. J., and Demuth, D. R. (2007). Differential interaction of Aggregatibacter (Actinobacillus) actinomycetemcomitans LsrB and RbsB proteins with autoinducer 2. J. Bacteriol. 189, 5559-5565.

Shrout, J. D., Chopp, D. L., Just, C. L., Hentzer, M., Givskov, M., and Parsek, M. R. (2006). The impact of quorum sensing and swarming motility on Pseudomonas aeruginosa biofilm formation is nutritionally conditional. Mol. Microbiol. 62, 1264-1277.

Shrout, J. D., Tolker-Nielsen, T., Givskov, M., and Parsek, M. R. (2011). The contribution of cell-cell signaling and motility to bacterial biofilm formation. MRS Bull. 36 , 367-373.
Surette, M. G., Miller, M. B., and Bassler, B. L. (1999). Quorum sensing in Escherichia coli, Salmonella typhimurium, and Vibrio harveyi: a new family of genes responsible for autoinducer production. Proc. Natl. Acad. Sci. U.S.A. 96, 1639-1644

Verhaegh, S. J., Snippe, M. L., Levy, F., Verbrugh, H. A., Jaddoe, V. W., Hofman, A., Moll, H. A., van Belkum, A., and Hays, J. P. (2011). Colonization of healthy children by Moraxella catarrhalis is characterized by genotype heterogeneity, virulence gene diversity and cocolonization with Haemophilus influenzae. Microbiology 157, 169-178.

Waters, C. M., and Bassler, B. L. (2005). Quorum sensing: cell-to-cell communication in bacteria. Annu. Rev. Cell Dev. Biol. 21, 319-346.

Conflict of Interest Statement: The author declares that the research was conducted in the absence of any commercial or financial relationships that could be construed as a potential conflict of interest.

Received: 07 April 2012; accepted: 02 July 2012; published online: 19 July 2012.

Citation: Swords WE (2012) Quorum signaling and sensing by nontypeable Haemophilus influenzae. Front. Cell. Inf. Microbio. 2:100. doi: 10.3389/fcimb. 2012.00100

Copyright () 2012 Swords. This is an open-access article distributed under the terms of the Creative Commons Attribution License, which permits use, distribution and reproduction in other forums, provided the original authors and source are credited and subject to any copyright notices concerning any thirdparty graphics etc. 